\title{
Operative sequentiality in tumor differentiation and progression as protein molecular structure and sequence context in modulating alternative splicing events
}

\author{
Lawrence M. Agius
}

Department of Pathology, Mater Dei Hospital, University of Malta Medical School, Msida, Malta

Email: lawrence.agius@um.edu.mt, lawrence.agius@gov.mt

Received 7 March 2013; revised 10 April 2013; accepted 15 May 2013

Copyright (C) 2013 Lawrence M. Agius. This is an open access article distributed under the Creative Commons Attribution License, which permits unrestricted use, distribution, and reproduction in any medium, provided the original work is properly cited.

\begin{abstract}
This review article discusses dimensional reconstruction of alternative splicing that not only affects primarily the distributional dimensions of isoforms of various protein species but especially influences the nature of interactivity events between various protein species and also the structure of the given protein molecules. In such terms, disorders of differentiation of individual tumors and of tumor types and subtypes would correlate with distinctive dimensions of expression of a limited number of genes in various modes of expressed selectivity programs. In particular, the differentiation programs of normal tissues would correlate with combinatorial systems of splicing factors and of auxiliary factors in the development of patterns of gene expression. The significance of mis-splicing events is consonant with the wide range of phenotypic expression of neoplastic lesions and in the great variety of differentiation patterns and also of the variable degrees of differentiation of various components of a given neoplasm. The structure of given protein isoforms resulting from alternative splicing correlate with the sequence context of exons in the enhancement or inhibition of splicing events and would also influence pathobiologic behavior patterns of given neoplastic lesions. The development of abnormal cell signalling pathways and of interactivity patterns in a combinatorial way would directly influence the stability and trafficking dynamics of given protein molecular species in inducing an abundance of protein isoform production. Series of multi-component systems ranging from receptivity to consequential pathways of development of differential phenotype would allow for a high degree of modulatory effect within systems implicating in particular the interactions of individual tumor cells with each
\end{abstract}

other and with the matrix components. It is within the context of constitutive versus alternative splicing events that this review article proposes that proportional recreation of differentiation pathways promotes a self-progression of the pathobiologic processes of a given neoplastic lesion.

Keywords: RNA Splicing; Neoplasms; Tumorigenesis; Protein Isoforms

\section{INTRODUCTION}

Pre-mRNA splicing incorporates both constitutive and alternative modes of generation of a wide variety of proteins within the context of a variety of disorders including in particular developmental processes and neoplastic disease. More than half of all alternative splicing events are regulated by multiple heterogeneous nuclear ribonucleoparticle proteins [1]. Relative to developmental equilibria in neogenesis, it is significant to consider the wide diversity of gene expression in the presence of a relatively small number of genes as a main contributor to molecular biology of the proteome. Dynamics of equilibrium implicate various isoforms of protein molecules that determine cell fate or differentiation attribute as these are expressed in tumorigenesis. Splicing of non-functional transcripts of the BRAF proto-oncogene affects overall cellular activity and possibly induces a decrease in growth signal sensitivity in colorectal cancer [2].

Alternative splicing may occur in the absence of mutation to given acceptor sequences that are bound to pre-mRNA molecules. Alternative splicing can implicate exon skipping and the expression of other isoforms of protein molecules. Such protein molecules may constitute enzymes, or the expression of pro-apoptotic or antiapoptotic molecules that promote or suppress survival of 
cells.

The machinery of splicing includes the multi-component spliceosome that binds to given sequences on the pre-mRNA in a manner that utilizes exonic or intronic enhancers or suppressors.

\section{PHENOTYPE EXPRESSION}

Phosphorylation also modulates alternative splicing by influencing the serine/arginine rich sequences on the SR molecule.

Determination of origin of phenotype expression in neoplasms is a highly likely result of alternative splicing of mRNA in conjunction with a number of other transcriptional, post-transcriptional and post-translational events in the diversification of the proteome.

Hence, it would appear that diversity of protein molecules does not arise directly due to variability of gene expression but is mediated by the generation of modulated mRNA molecules in alternative splicing. A melanoma-specific CD44 alternative splicing pattern shows quantitative variability during tumor progression but a qualitatively stable pattern [3]. Some $60 \%-70 \%$ of genes in the human genome undergo splicing and so the biological significance of splicing is inherently immense.

Neoplasia is a contributor to phenotypic variability in a manner that is unstable in terms of comparison with constitutive splicing of normal tissues or as expressed developmentally. Extensive changes in alternative splicing play a profound role in shaping the changes in cell behaviour that characterize epithelial-to-mesenchymal transition [4].

Suppressors and enhancers of alternative splicing are influenced by various chemicals or small molecules in a manner that affects alternative splicing. In such terms, the potentiality of regulation and suppression of tumorigenesis is considerable and would constitute a highly promising approach to chemotherapeutic management of neoplasms in general.

\section{VASCULAR ENDOTHELIUM}

Alternative splicing of vascular endothelial growth factor leads to several different isoforms, which are differentially expressed in various tumor types and have distinct functions in tumor blood vessel formation [5].

In addition, the recognition of disease or tumor markers as diagnostic markers and expressed therapeutic molecules constitutes potential resultant compounds in the management of patients with neoplasia. Osteopontin splice variants differentially affect clinicopathologic features and biology in gastric carcinoma [6].

Another aspect of alternative splicing is the influence of different isoforms in the intracellular trafficking of protein molecules, particularly the shuttling of such molecules between the nucleus and the cytoplasm. Alternative splicing of ribosomal S6 kinase 1 acts as a molecular switch in breast carcinoma cells, raising levels of oncogenic isoforms that activate mTORC1 [7].

It is particularly with regard to such modulated effect that the differential effects of alternative splicing can assume important regulatory functionality within various cellular types and subtypes. Versican V2 isoform proteoglycan enhances angiogenesis and also mediates upregulation of fibronectin in brain tumors [8].

The distinction of isoforms allows for the establishment of an equilibrium that quantitatively implicates various different isoforms of the same protein molecule. Enzymes in particular may respond in differential manner in cascade pathways and would therefore implicate a series of alternative outcomes to cell metabolic pathway processing. A pentaspan membrane glycoprotein prominin-1 (CD133) is a universal marker for cancer stem cells and splicing variants of CD133 are implicated in the complicated gene regulation in a context-dependent manner [9].

The creation of differential isoforms by splicing that affect suppressor genes and oncogenes would potentially modulate carcinogenesis itself in the generation or nongeneration of neoplasms.

Oncogenic splicing factor SRSF1 is a critical MYC target by enabling this to regulate the expression of specific protein isoforms through alternative splicing [10].

Constitutive realization of the spliced mRNA would allow for prevention or suppression of tumorigenesis of a potentially wide variety of tumor types.

Apoptosis depends on a balance between effects exerted by pro-apoptotic pathways and anti-apoptotic processes, and hence such approach utilizing alternative splicing events could allow for the therapeutic suppression of tumors by suppressing the life-span of the neoplastic cells.

Regulatory proteins may also be modulated therapeutically to control splicing events. The emergence of such modulation can be coupled to phosphorylation of protein molecules and also allow for protein:protein interactivity in the exchange of active splice site selectivity. Betacatenin regulates RNA alternative splicing and stability possibly by binding to target RNA in cells [11].

\section{ALTERNATIVE USAGE}

Alternative usage of exons on mRNA molecules may regulate in particular the phosphorylation state of regulatory proteins and allow for the exertion of differential modulatory effect and for variable concentration of regulatory proteins. Added to this is the flexibility of exonic regulatory sequences that allow for the emergence 
of a variety of protein molecular isoforms. Malignancyspecific gene expression differences occur within a pluripotent stem cell context [12].

Developmental processes rely on differential tissue concentration of small amounts of regulatory proteins and this would allow for the distinctive expression of alternative splicing events in phenotype expression of differentiated cell types both in the developmental setting and also in neoplasia.

Epithelial-mesenchymal transition (EMT) endows cells with invasive properties, inhibits apoptosis and sensecence, contributes to immunosuppression and induces stress resistance and stem cell properties. Transforming growth factor-Beta induces changes in alternative splicing to initiate and maintain EMT [13].

The use of various isoforms as preferential modes of expression of genes would hence contribute not only to the differential protein subtype determination but also to the constitutive versus alternative activity of the splicing machinery itself. The splicing factor SRSF6 up- or downregulates the splicing of several tumor suppressors and oncogenes and is an oncoprotein that controls proliferation and survival of lung and colon cancer cells [14].

\section{SPECIFIC SEQUENCES}

mRNA splicing includes the inclusion of specific sequences in the molecule by the spliceosome in a manner that may allow the utilization also of small interfering RNA molecules. Such an approach that employs small interfering RNAs would constitute a highly promising approach that potentially regulates the production of multiple different isoforms of the same protein molecule. Two splice variants from the Bcl-x gene via alternative 5' splice site selection are proapoptotic Bcl-x (s) and antiapoptotic Bcl-x (L) [15].

Splicing factors with rich serine/arginine sequences in the RS terminals cooperate with a series of combinatory factors that enhance or inhibit binding to the mRNA in selecting specific sequences in exonic splicing.

Such combinatory interactivity holds great promise in the treatment of neoplasms where mutations of the serine/arginine rich splicing factors or misplicing events may occur. Manipulation of alternative splicing as by antisense-mediated exon skipping is a unique way of regulating gene expression [16]. The administration of small molecule drugs may "mend" splicing factor pathways and allow for selection of specific sequences in the mRNA molecules.

Tumors may show enhanced metastatic potential or receptor binding abnormalities in the presence of mutations in SR sequences or in mis-splicing events. Receptor abnormalities may permit interactivity of tumor cells with matrix proteins and also interactions with adhesion molecules in enhancing spread of the tumor.

\section{SPLICING MODULATION}

The identity of constitutive versus alternative splicing events is a fundamental series of mechanisms that allows the differential usage of exons in addition to such phenomena as mRNA editing and post-translational pathways in protein: Protein interaction. Effective approaches in modulating splicing therapeutically include restoring open reading frames, influencing alternative splicing, and inducing exon inclusion [17].

Regulatory pathways are essential aspects of selective splicing sequence selection and can render protein molecules unstable or relatively susceptible to early molecular decay.

An essential combinatory series of interactions allows for the emergence of alternative splicing in neoplastic development that can skip exons or allow, conversely, great flexibility in sequence binding to splicing factors with no essential sequence specificity in binding required. Polarized and growth-arrested cellular architecture correlates with absence of alternative hMENA (an actin cytoskeleton regulator) isoform expression and may be of relevance in malignant progression to invasive cancer [18].

Mutational lesions can seriously impair sequence binding to splicing factors in a manner that promotes gene expression in abnormal patterns of mis-splicing pathways.

Combinatory pathways utilizing 5' end terminal U1 and 3' end terminal cis and trans factors would operate in alternative splicing events in inducing exonic usage or skipping.

\section{SPLICING COMPLEMENTING}

Multiple processes can complement splicing events and include editing of the mRNA molecule and various posttranslational events in inducing the production of many isoforms of protein molecules. Establishing the functional roles of genetic variants remains a significant challenge in the post-genomic era [19].

The high degree of selectivity in splicing of specific exons would apparently correlate with specific aspects of pathobiologic behavior and other attributes of neoplasms in a manner that promotionally advance understanding of progression of carcinogenesis and spread of tumor lesions.

Evidence suggests that the three structurally different GLI1 isoforms are distinctly different in their expression patterns and functions in the context of gliomas [20].

Such an ultimate aim would allow the determination of earlier diagnosis, prognosis and therapeutic intervention in patients with given tumor type and sub-type. 


\section{PHOSPHORYLATION}

Control of phosphorylation at RS terminals by phosphatases or topoisomerase I inhibitors would allow for the selectivity of splicing sequence binding to be modulated in desired modes in an attempt to inhibit tumorigenesis. Highly selective sequence binding holds the promise of a very low toxicity index in the treatment of such neoplastic lesions. Sodium butyrate is known to selectively mend sequences that otherwise give rise to mis-splicing events in carcinogenesis. Genes encoding splicing factors are among the most highly mutated in various haematological malignancies [21].

The multiple protein isoforms that may arise from expression of a given gene would account for a whole series of possible splicing events that combinatorily correlate with selectivity of exon usage or skipping.

The distinctive nature of alternative splicing versus constitutive splicing depends particularly on the presence of regulatory proteins that are present in given tissue types in generally small amounts. Further modification of protein isoform characterization will allow for the selectivity required in therapeutic modulation of neoplasms and their micro-environment, since alterations of the splicing machinery can cause the development of neoplasms [22].

Long non-coding RNAs are involved in alternative splicing of pre-m RNA, chromatin regulation, nuclear organization, genomic imprinting, and dosage compensation [23].

An interesting aspect of splicing events is the apparent close correlation of constitutional development conditioning with neoplastic evolution in terms of splicing and mis-splicing events. The glioma-associated oncogene homolog 1 (GLI1) family of zinc finger transcription factors is the nuclear mediator of the Hedgehog pathway in tumor development and progression [24]. In such terms, an overall series of system pathways would particularly affect interactivity with the surrounding matrix environment and as receptor biology leading eventually to metastatic spread of tumors.

\section{DESTABILIZATION}

Destabilization of protein isoforms allows for the controlled decay of protein molecules that allow for further conformational interference by such molecules as small interfering RNAs.

Cyclical activity as phosphorylation and dephosphosphorylation are recognized source for variability in activity of splicing factors by involving the 3' N-terminal of these factors in the RS sequences.

Mis-splicing events can also introduce stop condons or frameshift alterations in the mRNA molecules in a manner that directly affects expression of given protein iso- forms.

In such events, the patterns of gene expression would constitute a marker for the realization of given neoplasms or other disorders such as neurological disease states including muscular dystrophy.

Requirements in the operability of events would constitute a series of recognition motifs in the 3' N-terminal in particular and also the specific sequences in the 5' serine/arginine carboxyl terminal of splicing factors. Such distributional, differential pathways include the interactivity of combinatory pathways involving enhancing and inhibitory exonic sequences in mRNA and intronic sequences within given genes.

\section{TUMOR EVOLUTION}

Tumor lesions allow for the evolutionary development and expression of isoforms that cooperatively promote or inhibit gene expression within sequence contexts involving protein isoforms.

The emergence of a whole series of protein isoforms from the expression of a single specific gene would conform to the establishment of lesions that specifically operate as interactivity with other tumor or parenchymal/ stromal cells or with matrix components.

Both splicing regulation and epigenetic factors contribute to neoplastic progression, but the links between these two modes of gene-expression regulation in pathogenesis are poorly understood [25].

Integrative phenomena allow for the expression of genes that are closely allied with abnormalities or other attributes of intracellular trafficking. The expression of protein isoforms is significantly linked with the degree of activity or interaction of the involved protein isoforms with other proteins and also with such phenomena as sequestration within given sites in the cells affected.

Also, the degree of stability of protein molecular species is influenced by abnormal intracellular trafficking and affects such primary processes as cell signalling pathways.

The spliceosome itself constitutes a large complex of splicing factors and of heterogeneous ribonucleoproteins and also non-ribonucleoprotein moieties in selectively targeting given sequences in the mRNA.

Possible mutations in the spliceosome may also contribute to mis-splicing events and lead to or be closely related to neoplastic development.

Protein:RNA and protein:protein interactions are influenced by mis-splicing or alternative splicing patterns and further promote the potential realization of neoplastic progression. Several lines of evidence point to increased RNA-binding ability of polypyrimidine tractbinding protein-associated splicing factor and aberrant expression of long non-coding RNAs are related to human neoplasia [26]. 
In general, the overall dimensions for selectivity of sequence binding on mRNA molecular species require selectivity in the choice and targeting of constitutive or alternative splicing events in the prevention of multiple disorders including neoplasia. As in the breast cancer transcriptome, splicing is critical for the cell to "tailormake” specific functional transcripts [27].

\section{CONCLUDING REMARKS}

The dimensions of selectivity in sequence binding and in the institution of expression by a limited number of genes allow for the production of a series of protein isoforms that may in turn constitute multiple molecular species of distinct nature and structure.

The therapeutic modulation of lesions in splicing pathways would require a high degree of sequence selectivity with a given sequence context in its own right. It is towards the performance of realization of such phenomena as cell signalling and cell receptivity that alternative splicing events fundamentally can modulate and otherwise influence the pathobiology of tumor growth and spread.

The developmental correlative systems in any given tissue would indicate or otherwise aid in the recognition of regulatory factors that influence splicing events and the institution of multiple possible alternative splicing programs as contrasted with constitutional splicing events perse.

\section{REFERENCES}

[1] Huelga, S.C., Vu, A.Q., Arnold, J.D., Liang, T.Y., Liu, P.P., Yan, B.Y., et al. (2012) Integrative genome-wide analysis reveals cooperative regulation of alternative splicing by hn RNP proteins. Cell Reports, 1, 167-178. doi:10.1016/j.celrep.2012.02.001

[2] Hirschi, B. and Kolligs, F.T. (2013) Alternative splicing of BRAF transcripts and characterization of C-terminally truncated B-Raf isoforms in colorectal cancer. International Journal of Cancer, 133, 590-596. doi:10.1002/ijc.28061

[3] Raso-Barnett, L., Banky, B., Barbai, T., Becsagh, P., Timar, J. and Raso, E. (2013) Demonstration of a melanoma-specific CD44 alternative splicing pattern that remains qualitatively stable, but shows quantitative changes during tumor progression. PLoS One, 8, e53883. doi:10.1371/journal.pone.0053883

[4] Warzecha, C.C. and Carstens, R.P. (2012) Complex changes in alternative pre-m RNA splicing may play a central role in the epithelial-to-mesenchymal transition (EMT). Seminars in Cancer Biology, 22, 417-427. doi:10.1016/j.semcancer.2012.04.003

[5] Finley, S.D. and Popel, A.S. (2012) Predicting the effects of anti-angiogenic agents targeting specific VEGF isoforms. AAPS Journal, 14, 500-509.

[6] Tang, X., Li, J., Yu, B., Su, L., Yu, Y., Yan, M., et al.
(2013) Osteopontin splice variants differentially exert clinicopathological features and biological functions in gastric cancer. International Journal of Biological Sciences, 9, 55-66. doi:10.7150/ijbs.5280

[7] Ben-Hur, V., Denichenko, P., Siegfried, Z., Maimon, A., Krainer, A., Davidson, B., et al. (2013) S6K1 alternative splicing modulates its oncogenic activity and regulates mTORC1. Cell Reports, 3, 103-115. doi:10.1016/j.celrep.2012.11.020

[8] Yang, W. and Yee, A.J. (2013) Versican V2 isoform enhances angiogenesis by regulating endothelial cell activities and fibronectin expression. FEBS Letters, 587, 185192. doi:10.1016/j.febslet.2012.11.023

[9] Tabu, K., Bizen, N., Taga, T. and Tanaka, S. (2013) Gene regulation of prominin-1 (CD133) in normal and cancerous tissues. Advances in Experimental Medicine and Biology, 777, 73-85. doi:10.1007/978-1-4614-5894-4_5

[10] Das, S., Anczukow, O., Akerman, M. and Krainer, A.R. (2012) Oncogenic splicing factor SRSF1 is a critical transcriptional target of MYC. Cell Reports, 1, 110-117. doi:10.1016/j.celrep.2011.12.001

[11] Kim, I., Kwak, H., Lee, H.K., Hyun, S. and Jeong, S. (2012) Beta-catenin recognizes a specific RNA motif in the cyclooxygenase-2 m RNA 3'-UTR and interacts with HUR in colon cancer cells. Nucleic Acids Research, 40, 6863-6872. doi:10.1093/nar/gks331

[12] Alagaratnam, S., Harrison, N., Bakken, A.C., Hoff, A.M., Jones, M., Sveen, A., et al. (2013) Transforming pluripotency: An exon-level study of malignancy-specific transcripts in human embryonal carcinoma and embryonic stem cells. Stem Cells and Development, 22, 1136-1146. doi:10.1089/scd.2012.0369

[13] Saitoh, M. and Miyazawa, K. (2012) Transcriptional and post-transcriptional regulation in TGF-Beta-mediated epithelial-mesenchymal transition. The Journal of Biochemistry, 151, 563-571. doi:10.1093/jb/mvs040

[14] Cohen-Eliav, M., Golan-Gerstl, R., Siegfried, Z., Andersen, C.L., Torsen, K., Orntoft, T.F., et al. (2012) The splicing factor SRSF6 is amplified and is an oncoprotein in lung and colon cancers. The Journal of Pathology, 229, 630-639.

[15] Schultz, J.C., Vu, N., Shultz, M.D., Mba, M.U., Shapiro, B.A. and Chalfant, C.E. (2012) The proto-oncogene PKC1 regulates the alternative splicing of Bcl-x Pre-m RNA. Molecular Cancer Research, 10, 660-669. doi:10.1158/1541-7786.MCR-11-0363

[16] Wan, J. (2012) Antisense-mediated exon skipping to shift alternative splicing to treat cancer. Methods in Molecular Biology, 867, 201-208. doi:10.1007/978-1-61779-767-5_13

[17] Spitali, P. and Aartsma-Rus, A. (2012) Splice modulating therapies for human disease. Cell, 148, 1085-1088. doi:10.1016/j.cell.2012.02.014

[18] Di Modugno, R., Iapicca, P., Boudreau, A., Mottolese, M., Terrenato, I., Perracchio, L., et al. (2012) Splicing program of human MENA produces a previously undescribed isoform associated with invasive, mesenchymal-like breast tumors. Proceedings of the National Academy of 
Sciences of the United States of America, 109, 1928019285. doi:10.1073/pnas.1214394109

[19] Li, G., Bahn, J.H., Lee, J.H., Peng, G., Chen, Z., Nelson, S.F., et al. (2012) Identification of allele-specific alternative m RNA processing via transcriptome sequencing. Nucleic Acids Research, 40, e104. doi:10.1093/nar/gks280

[20] Carpenter, R.L. and Lo, H.W. (2012) Identification, functional characterization and pathobiological significance of GLI1 isoforms in human cancers. Vitamins \& Hormones, 88, 115-140.

[21] Bonnal, S., Vigevani, L. and Valcarcel, J. (2012) The spliceosome as a target of novel antitumour drugs. Nature Reviews Drug Discovery, 11, 847-859. doi:10.1038/nrd3823

[22] Miura, K., Fujibuchi, W. and Unno, M. (2012) Splice isoforms as therapeutic targets for colorectal cancer. Carcinogenesis, 33, 2311-2319. doi:10.1093/carcin/bgs347

[23] Niland, C.N., Merry, C.R. and Khalil, A.M. (2012) Emerging roles for long non-coding RNAs in cancer and neurological disorders. Frontiers in Genetics, 3, 25. doi:10.3389/fgene.2012.00025

[24] Carpenter, R.L. and Lo, H.W. (2012) Hedgehog pathway and GLI1 isoforms in human cancer. Discovery Medicine, 13, 105-113.

[25] Dardenne, E., Pierredon, S., Driouch, K., Gratadou, L., Lacroix-Triki, M., Espinoza, M.P., et al. (2012) Splicing switch of an epigenetic regulator by RNA helicases promotes tumor-cell invasiveness. Nature Structural \& Molecular Biology, 19, 1139-1146. doi:10.1038/nsmb.2390

[26] Wu, C.F., Tan, G.H., Ma, C.C. and Li, L. (2013) The non-coding RNA llme23 drives the malignant property of human melanoma cells. Journal of Genetics and Genomics, 40, 179-188. doi:10.1016/j.jgg.2013.03.001

[27] Eswaran, J., Horvath, A., Godbole, S., Reddy, S.D., Mudvari, P., Ohshiro, K., et al. (2013) RNA sequencing of cancer reveals novel splicing alterations. Scientific Reports, 3, Article ID: 1689. doi:10.1038/srep01689 